\title{
Antagonistic activity of the native strain of Pseudomonas chlororaphis 3 of the Belgorod region against the bacterium of the genus Ralstonia
}

\author{
Alina Avakova ${ }^{1 *}$, Nikita Lyakhovchenko ${ }^{1}$, Roman Lopin ${ }^{1}$, Elizaveta Goltseva ${ }^{1}$, Maria \\ Belozerskikh ${ }^{1}$, and Inna Solyanikova ${ }^{1,2}$. \\ 1 - Belgorod State National Research University, 308015, Belgorod, Russia. \\ 2 - G.K. Skryabin IBPM PSCBR RAS, 142290, Moscow, Russia.
}

\begin{abstract}
The paper presents data on the evaluation of the antagonistic potential of Pseudomonas chlororaphis 3 and P. chlororaphis BS-1393 strains against the bacterium of the genus Ralstonia. It was revealed that the native strain is $30 \%$ more effective than the collection one. A scheme for obtaining an ethyl acetate extract of phenazine compounds, whose antibacterial activity exceeds the effectiveness of the extractant (ethyl acetate) by $57 \%$, has been developed.
\end{abstract}

Keywords: Pseudomonas chlororaphis 3, antagonistic activity, phenazine.

\section{Introduction}

The bacteria of the genus Ralstonia are the causative agents of bacterial wilting of the most important crops, such as potatoes, tomatoes, peppers, eggplants and others. It is a typical soil inhabitant, found both on cultivated soils and on virgin ones. High soil moisture and periods of rainy weather (especially at elevated temperatures) create favorable conditions for the development of phytopathogen. The ability of Ralstonia to infect a large number of hosts makes it difficult to control the infectious process. Based on this, to date, no method of combating the causative agent of the disease is sufficiently effective [1]. As an alternative to fungicides or as an additional method in the complex of plant protection measures, the potential of natural antagonists of phytopathogenic fungi and bacteria - non-pathogenic microorganisms that saprotrophically live on plant residues in the soil, develop in the rhizosphere, phyllosphere or endophytic in plants that compete with phytopathogens, inhibiting their development, and can also have a positive effect on plants-has been actively investigated in the last decade [2]. As a result, one of the participants in such interactions gets a selective advantage in the struggle for survival due to competitive properties: the production of antibiotic substances, high growth and adaptive capabilities [3].

*Author: 1378405@bsu.edu.ru 
The aim of this work is to evaluate the antagonistic activity of the native strain of the Belgorod region Pseudomonas chlororaphis 3 [4] against a representative of the genus Ralstonia.

\section{Materials and methods}

Determination of the antagonistic properties of $P$. chlororaphis strain 3 against the Ralstonia bacterium was determined by the agar disk method. As a control culture, the $P$. chlororaphis strain BS-1393, a component of the biopreparation "Pseudobacterin-2", was used. Suspensions of $P$. chlororaphis strain 3 and P. chlororaphis BS-1393 bacteria equal to 0.5 optical units (OU) at a wavelength of $\lambda=600 \mathrm{~nm}$ (Microscan Turbidity Meter, Siemeps, USA) were sown with a "lawn" of $100 \mu \mathrm{l}$ in pre-prepared Petri dishes with a nutrient medium (composition (g/l): $3 \%$ peptone, $1000 \mathrm{ml}$ tap water). The crops were incubated for two days. Then, with a sterile cork drill (diameter $9 \mathrm{~mm}$ ), agar blocks were cut out of the medium layer and transferred to the surface of the agarized medium, which had just been inoculated with a suspension of the test culture of the genus Ralstonia, equal to 0.5 OD. The exposure time was 18 hours at a temperature of $25^{\circ} \mathrm{C}$. The average radius of the suppression zones of the growth of the "lawn" of the phytopathogen was used to judge the degree of antagonistic activity of the studied pseudomonads.

To obtain phenazine compounds (Fig. 1), the culture of $P$. chlororaphis 3 was cultured in $3 \%$ liquid peptone $(250 \mathrm{ml})$ for 18 hours $\left(\right.$ at $\left.25^{\circ} \mathrm{C}\right)$. The resulting inoculate was loaded into a bioreactor (Biocanvas LFDV1,5L, Centrion, Korea) containing $250 \mathrm{ml}$ of the same nutrient medium and cultured for 3 days at $25^{\circ} \mathrm{C}$ and $\mathrm{pH}=7$. Aeration was at the level of 0.05 PLS. The mixing mode varied from 0 (for 12 hours) to $150 \mathrm{rpm}$ (12 hours). This was due to the fact that the biosynthesis of phenazines is associated with the quorum sensing mechanisms implemented during the formation of a biofilm at the interface.

After cultivation, the culture liquid was separated from the biomass by centrifugation (using a Neofuge 1600R centrifuge, Heal Force, China) at $4500 \mathrm{~g}$ for 20 minutes and the supernatant was taken. The sediment was filtered and dried at $100^{\circ} \mathrm{C}$ to a constant mass.

During the extraction of phenazines from the culture liquid, it was acidified with $2 \mathrm{~N}$ hydrochloric acid to $\mathrm{pH} \mathrm{1-2} \mathrm{and} \mathrm{ethyl} \mathrm{acetate} \mathrm{was} \mathrm{added} \mathrm{in} \mathrm{a} \mathrm{ratio} \mathrm{of} \mathrm{1:1.} \mathrm{The} \mathrm{mixture} \mathrm{was}$ thoroughly shaken for 30 minutes and the upper fraction was selected, then the extract was evaporated using a vacuum rotary evaporator (N-1000R). The concentration of phenazines was determined spectrophotometrically (UV-1900i, Shimadzu, Japan) in the wavelength range $\lambda=340-430$, in terms of phenazic acid, using the formula 1 :

$$
C=M \times \frac{A}{\varepsilon \times l},
$$

where $C$ is the concentration of the solution, $\mathrm{g} / \mathrm{l} ; \varepsilon$ is the molar absorption coefficient (for PCA, $\left.\varepsilon_{367}=3019 \mathrm{~mol}^{-1}\right) ; l$ is the thickness of the absorbing layer, $\mathrm{cm}(1 \mathrm{~cm}) ; A$ is the optical density of the absorbing substance; $M$ is the molar mass, $\mathrm{g} / \mathrm{mol}(224.21 \mathrm{~g} / \mathrm{mol})$ [5].

The antagonistic activity of the ethyl acetate extract against the bacteria of the genus Ralstonia was determined by the disc-diffuse method, which is based on the ability of the studied substances to diffuse from the paper disks impregnated with them into a solid nutrient medium, inhibiting the growth of microorganisms [6]. To conduct the study, a suspension of the Ralstonia with OD equal to $0.5 \mathrm{OU}$ at a wavelength of $\lambda=600 \mathrm{~nm}$, which was measured using a Microscan Turbidity Meter (manufactured by Siemeps, USA), was sown with a "lawn" of $100 \mu \mathrm{l}$ in pre-prepared Petri dishes with a nutrient medium. Paper sterile disks (9 $\mathrm{mm}$ in diameter), impregnated with $40 \mu \mathrm{l}$ of the test substance, were placed in seeded cups (6 disks per cup). Ethyl acetate (extractant) was used as a control. Antagonistic activity was judged by the average suppression radius of Ralstonia sp.

The significance of the differences was calculated by the difference method [7]. 


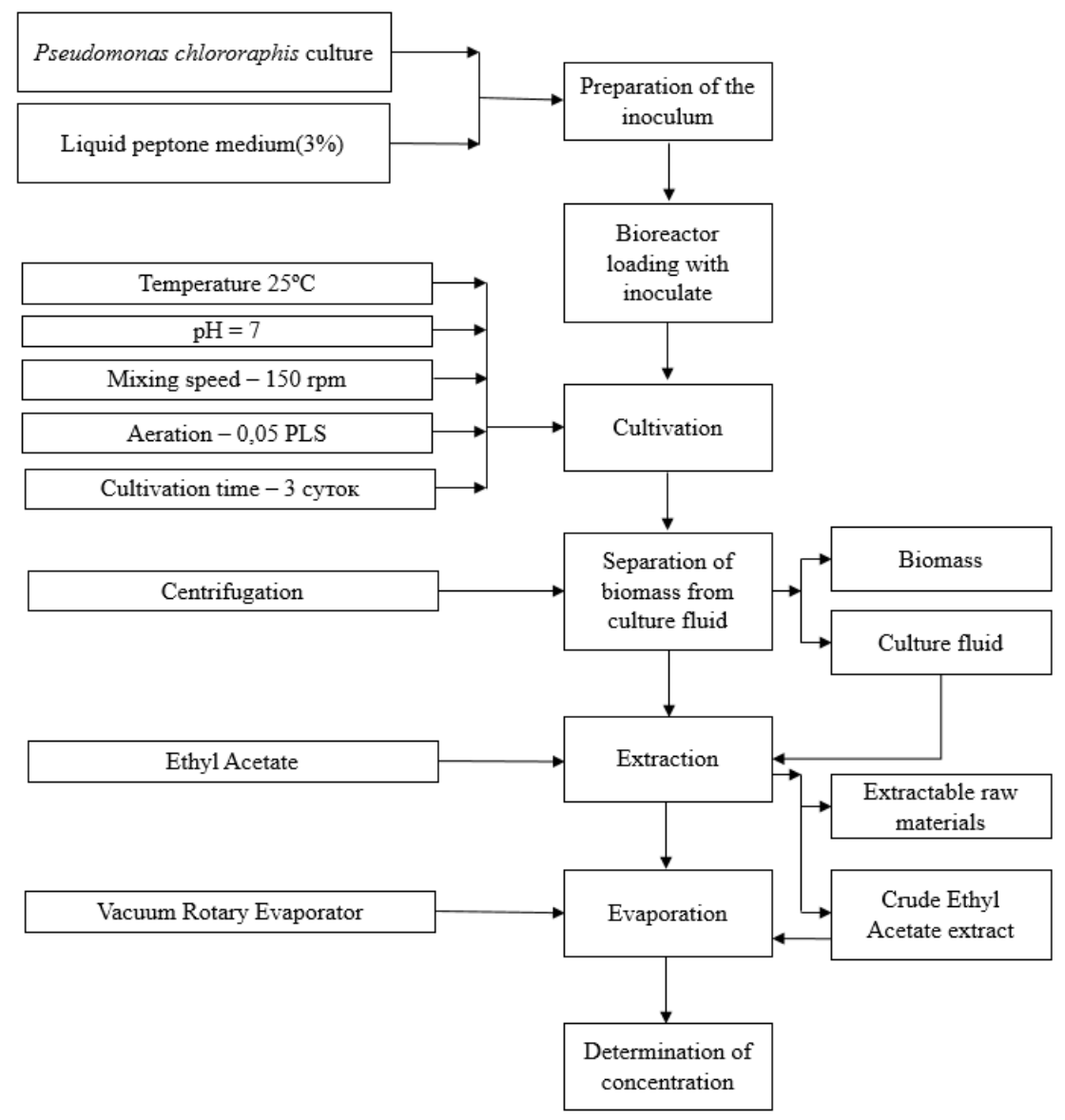

Fig. 1. General scheme for the preparation of phenazine compounds from the culture fluid of the producer of Pseudomonas chlororaphis 3.

\section{Results and discussion}

In the course of studying the antagonistic activity of Pseudomonas strains against Ralstonia sp. the agar disk method revealed that the effectiveness of $P$. chlororaphis strain 3 is statistically significantly higher P. chlororaphis BS-1393, since the average radius of phytopathogen suppression by native bacteria is $30 \%$ higher than that of the collection bacterium (Fig. 2), and the calculated Student confidence criterion $(\mathrm{t}=3.1)$ exceeded the tabular one (tst $=2.719)$ at an error level of $\mathrm{p}<0.01$.

It is known that P. chlororaphis bacteria have the ability to synthesize phenazines. Compounds of the phenazine series are a yellow crystalline substance and exhibit a high fungicidal effect and antimicrobial activity against Gram-positive and Ggram-negative bacteria, as well as protozoa [5].

The $P$. chlororaphis strain 3 was selected as a phenazine producer to produce this secondary metabolite. 


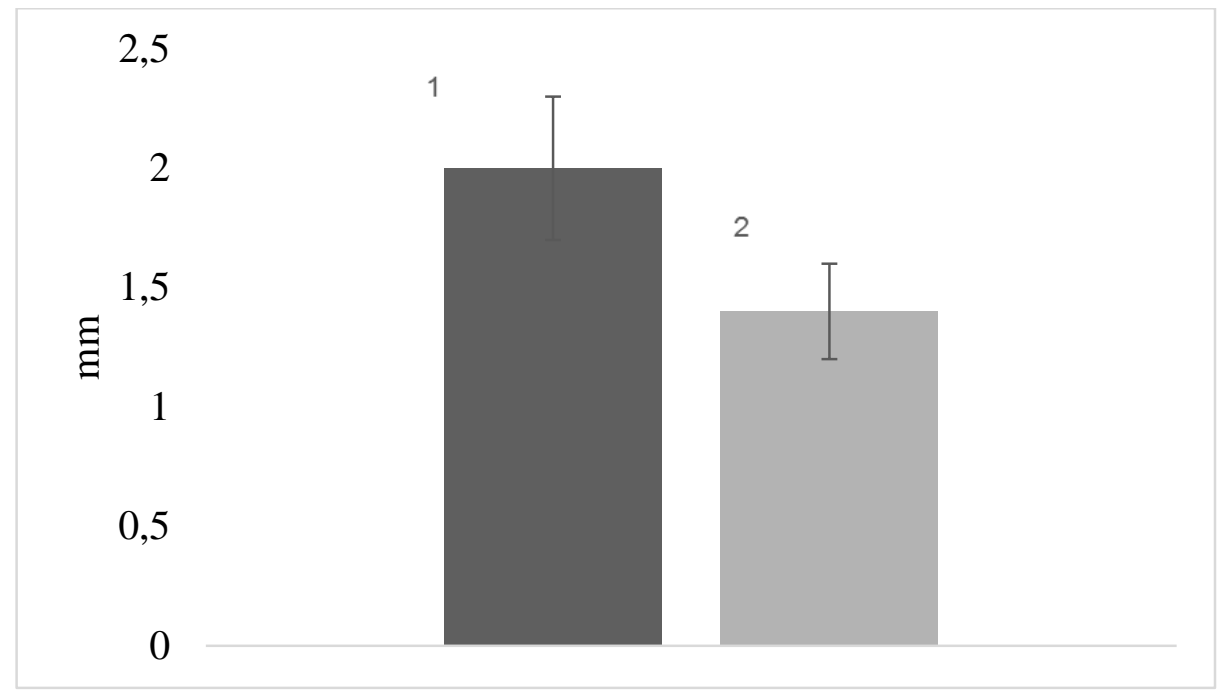

Fig. 2. The average radius of the growth suppression zone of the culture Ralstonia sp. as an indicator of the antagonistic activity of the bacteria Pseudomonas chlororaphis 3 (1) and Pseudomonas chlororaphis BS-1393 (2).

In the course of the work, a scheme for obtaining phenazines by biotechnological means was developed (Fig. 1). Spectrophotometric analysis of the evaporated ethyl acetate extract of the culture liquid showed a maximum absorption at $362 \mathrm{~nm}$ (Fig.3), which is close to the known value for phenazic acid [5]. The concentration of the metabolite in the extract was $5.4 \mathrm{mg} / 1$ (the dry weight of the biomass was $8.5 \mathrm{mg} / 1$ ).

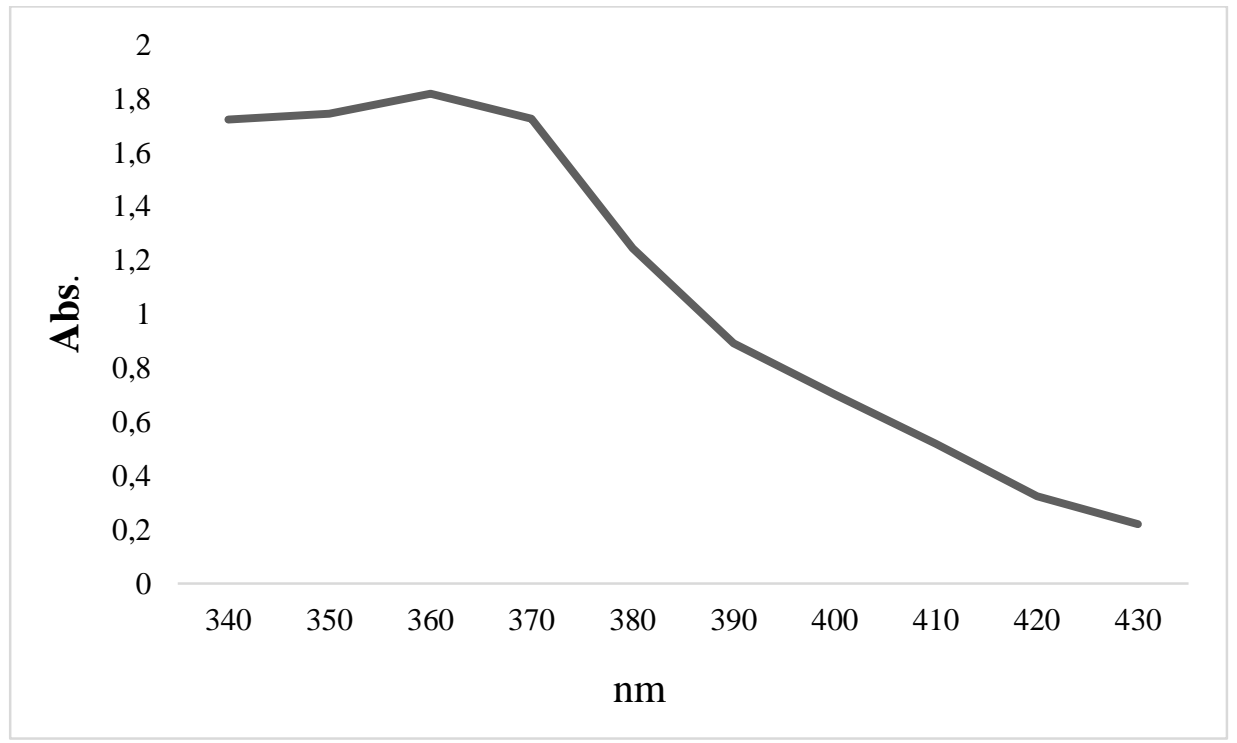

Fig. 3. Maximum absorption of ethyl acetate extract of culture liquid at $362 \mathrm{~nm}$ based on the result of spectrophotometric analysis in the wavelength range $\lambda=340-430 \mathrm{~nm}$.

The study revealed that the antibacterial activity of the ethyl acetate extract of phenazines exceeds the effectiveness of the extractant (ethyl acetate) by $57 \%$ (Fig. 4), while the calculated Student confidence criterion $(t=8.74)$ exceeded the tabular one $\left(t_{s t}=3.582\right)$ at an error level of $\mathrm{p}<0.001$. 


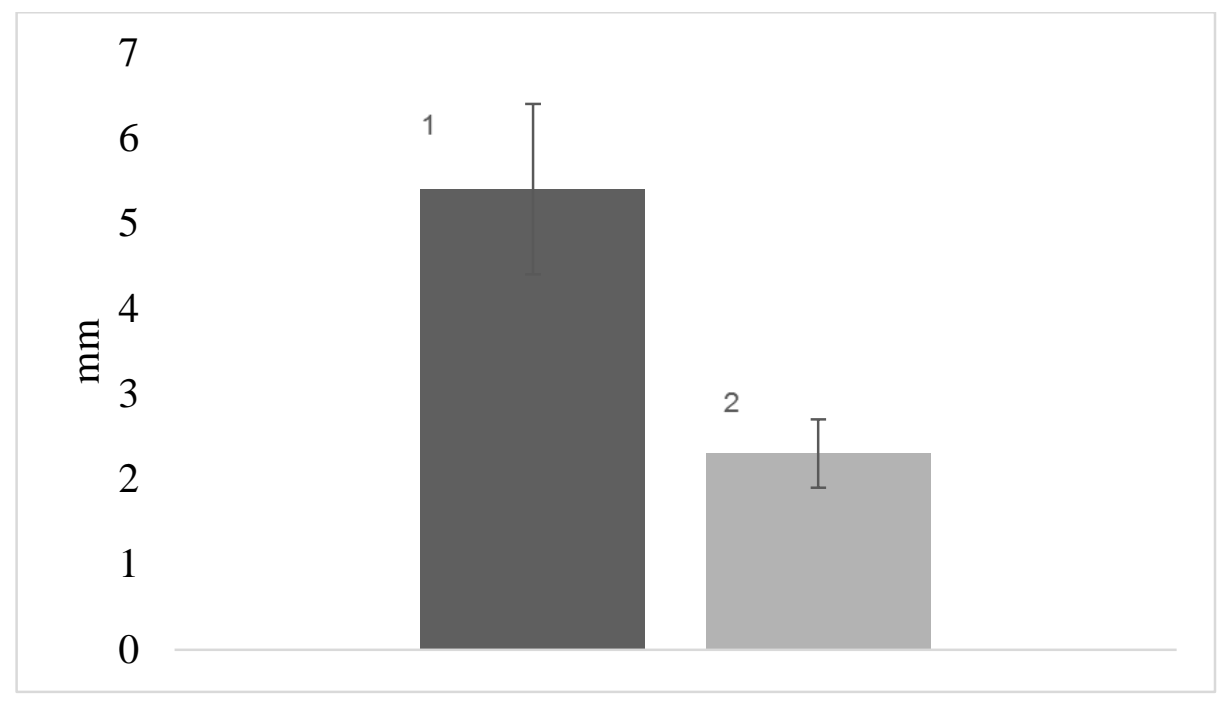

Fig. 4. The average radius of the growth suppression zone of the culture Ralstonia sp. as an indicator of the antifungal activity of ethyl acetate extract of phenazine compounds (1) and ethyl acetate (2).

Thus, it was found that the antagonistic activity of $P$. chlororaphis strain 3 against the bacteria of the genus Ralstonia is higher than that of $P$. chlororaphis BS-1393. In the course of cultivation of an indigenous bacterium, a substance with significant antimicrobial activity was obtained.

\section{Conclusion}

It is known that the use of bacteria and their secondary metabolites as components of biological plant protection products is widespread [8]. However, the antagonistic activity of bacterial strains of the same genus and species may vary. During the study, this was confirmed: the native bacterium P. chlororaphis 3, isolated from the soil of the Belgorod region, showed activity 30\% higher than the effectiveness of $P$. chlororaphis BS-1393 against Ralstonia sp.

Evaluation of the antagonistic activity of the ethyl acetate extract of phenazine compounds showed that the $P$. chlororaphis strain 3 can be a promising producer of antibiotic substances.

Funding: This research was funded by RFBR according to the research project № 19-5480003.

\section{References}

1. K.P. Kornev and others, Russian Agricultural Science Review, 6, 13 (2015).

2. G. D. Sokolova, A. P. Glinushkin, Mycology and phytopathology, 51, 191 (2017).

3. A. V. Semenov, Bulletin of the Orenburg scientific center, Ural branch, Russian Academy of Sciences, 1 (2013)

4. T. Z. Esikova, T. O. Anokhina, T. N. Abashina, N. E. Suzina, I. P. Solyanikova, Microorganisms, 9 (2021)

5. T. A. Rabina, Dissertation abstract for a bachelor's degree in biotechnology (19.03.01) NITPUTomsk, 67 (2016) 
6. I. A. Dudka, S. P. Wasser, I. A. Ellanskaya, Reference book (Naukova Dumka Publishing House-Kiev, 1982)

7. A. I. Netrusov, M. A. Egorova, L. M. Zakharchuk, Practicum on microbiology: Textbook. Manual for students higher studies, 608 (2005)

8. A. Pérez-García, D. Romero, A. De Vicente, Current opinion in biotechnology, 22, 187 (2011). 